\title{
Prevalence of Behavioural Risk Factors for Non-communicable Diseases Among Adolescents in Schools in Benin in 2016
}

\author{
Agbohoui Houinato Olga ${ }^{1, *}$, Hounkpatin Benjamin², Houehanou Yessito Corine Nadege1, \\ Kpozehouen Alphonse ${ }^{3}$, Gbedji Kevin Yann ${ }^{1}$, Vodougnon Charlemangne ${ }^{1}$, Guedou Fernand ${ }^{4}$, \\ Houinato Dismand Stephan ${ }^{1}$, Perrin Rene Xavier ${ }^{2}$ \\ ${ }^{1}$ Epidemiologic Laboratory for Chronic and Neurological Diseases (LEMACEN), University of Abomey Calavi, Cotonou, Benin \\ ${ }^{2}$ University Hospital of Mother and Child Lagune (CHU MEL), University of Abomey Calavi, Cotonou, Benin \\ ${ }^{3}$ Regional Institute of Public Health (IRSP), University of Abomey Calavi, Cotonou, Benin \\ ${ }^{4}$ Health Center of Sexually Transmitted Infections (DIST), Health Center of Cotonou1, Cotonou, Bénin
}

\section{Email address:}

oagbohoui@gmail.com (A. H. Olga), bhounkpatin@yahoo.fr (H. Benjamin), corinehouehanou@gmail.com (H. Y. C. Nadège), alphonse.kpozehouen@gmail.com (K. Alphonse), gbyann@gmail.com (G. K. Yann), charvodji@gmail.com (V. Charlemangne), guedaf@yahoo.fr (G. Fernand), dshouinato@gmail.com (H. D. Stéphan), perrinx@yahoo.fr (P. R. Xavier)

${ }^{*}$ Corresponding author

\section{To cite this article:}

Agbohoui Houinato Olga, Hounkpatin Benjamin, Houehanou Yessito Corine Nadege, Kpozehouen Alphonse, Gbedji Kevin Yann, Vodougnon Charlemangne, Guedou Fernand, Houinato Dismand Stephan, Perrin René Xavier. Prevalence of Behavioural Risk Factors for Non-communicable Diseases Among Adolescents in Schools in Benin in 2016. Science Journal of Public Health.

Vol. 7, No. 6, 2019, pp. 214-224. doi: 10.11648/j.sjph.20190706.16

Received: September 22, 2019; Accepted: October 15, 2019; Published: November 27, 2019

\begin{abstract}
Introduction: Non-communicable diseases (NCDs) are on the rise in sub-Saharan Africa. The overall objective of this study was to estimate the prevalence of behavioural risk factors for NCDs among adolescents in schools in Benin in 2016. Methods: This was a cross-sectional study conducted in 2016 and involving 3,841 adolescents of 10 to 19 years from 40 secondary schools in Benin. Participants were selected using a 2-level random sampling technique. Data were collected through a self-administered questionnaire and were analysed using Stata software. Associated factors were investigated by a logistic regression. Results: The average age of the participants was $16.54 \pm 0.04$ and the sex ratio was 1.16 . The prevalence of alcohol consumption was $48.3 \%$, and was associated with residence and class. The prevalence of tobacco use was $8 \%$, and was associated with gender, age, type of facility and residence. The prevalence of insufficient physical activity was $56.4 \%$, and was associated with gender. The prevalence of physical inactivity was $12.4 \%$, and was associated with age, class, socio-economic level of parents and type of facility. The consumption of soft drinks was $34.9 \%$, and was associated with gender and type of establishment. The prevalence of under-consumption of fruit and vegetables was $94.3 \%$. That of consumption of fast food was $38.6 \%$, and was associated with the type of establishment, the class and the person who the adolescent lived with. Conclusion: This work shows the importance of NCDs' Risk Factors in schools. It is now urgent to implement specific interventions in order to reverse the trend.
\end{abstract}

Keywords: Adolescents, Risk Factors, Non-communicable Diseases, School Environment, Benin

\section{Introduction}

Non-Communicable Diseases (NCDs) are responsible for 40 million deaths worldwide in $2017 ; 80 \%$ of which are in emerging countries [1]. According to the WHO estimates,
NCDs are expected to be the cause of the largest increase in deaths by the year 2020 [2]. Indeed, most emerging countries are undergoing an epidemiological transition trough the reduction of infectious diseases, increased life expectancy, urbanization and the adoption of the modern lifestyle of large 
cities [3]. This epidemiological transition is accompanied by a nutritional and social transition with the increase in alcohol and tobacco consumption and the abandonment of traditional meals for meals rich in sugars, meat and animal fats. This leads to the occurrence of chronic or degenerative diseases called "diseases of civilization" $[4,5]$. Thus, mortality due to NCDs in emerging countries has increased, and some other diseases such as cancers and diabetes unknown to the populations are taking on increasingly alarming proportions [3].

Although the management of the NCDs is costly for emerging countries, it is possible to take actions by addressing the risk factors (RFs) as early as possible, even with limited resources.

Sub-Saharan Africa is the region with the most alarming level NCDs in terms of adolescent and youth health, regarding particularly the importance of NCD risk factors which are in a steady upward trend [6]. Policies and interventions have been put in place with monitoring indicators to support adolescents and young people, but they are most often focused on reproductive health [6].

The 2009 Global School-based Student Health Survey (GSHS) in Benin revealed that: $20.7 \%$ of adolescents have consumed alcohol at least once; $3.7 \%$ have smoked cigarettes at least once during the 30 days preceding the survey; $1.9 \%$ have consumed cannabis during the 30 days preceding the survey; only 19\% normally have eaten fruits and vegetables, while $17.6 \%$ have eaten at least once in a fast food restaurant [7]. Those data provide evidence for the rise of NCDs' Risk Factors among adolescents in Benin.

It is within this framework that the present study, whose objective was to determine the prevalence of behavioural risk factors of the NCDs in schools in Benin and the factors associated with them, was conducted.

\section{Methods}

\subsection{Description of the Study and of the Study Population}

We analysed data from the national survey on the current status and factors associated with adolescent health in schools in Benin in 2016. This was a cross-sectional study targeting 10 to 19-year-olds children of both sex and enrolled from first to seventh (final) grades of secondary school. The number of subjects required was calculated by the Schwartz formula and was 3,841 . The sampling was conducted with the technical support of CDC Atlanta, using a 2-level random stratified sampling technique with a probability rate proportional to the size of the schools and classes.

The first level consisted in selecting 40 out of 1494 public and private secondary schools, with a probability rate proportional to the size of the institutions. At the second level, within each selected institution, a census of all classes was conducted. The choice of classes to be investigated was randomly made from a list of random numbers, preestablished and made available by CDC Atlanta and the WHO. This list of numbers varied from one school to another. The number of classes to be surveyed per institution was proportional to the total number of classes available in those selected institutions. Finally, all adolescents of eligible classes present during the survey were included in the study.

A standardized questionnaire was used for data collection. Investigation administrators trained after the informed consent of adolescents or their parents, guided them to fill in the questionnaire.

\subsection{Variables}

\subsubsection{Dependent Variables}

Dependent variables were: (1) alcohol consumption defined as alcohol consumption at least once in the last 30 days preceding the survey; (2) tobacco and cannabis consumption defined as consumption at least once in the last 30 days preceding the survey; (3) Insufficient physical activity defined as less than 60 minutes per day of moderate to sustained physical activity and less than 03 sessions of moderate to sustained physical activity per week; (4) Sedentariness defined as low energy use combined with sitting or half-lying for more than 4 hours per day; (5) insufficient consumption of fruits and vegetables defined as consumption of less than 400 grams or less than 5 intakes of fruit and vegetables per day during the last 30 days preceding the survey; (6) consumption of soft drinks or sweetened soft drinks defined as consumption of at least one soda during the last 30 days preceding the survey; (7) consumption of fast food defined by the intake of fast food such as cafeteria meals more than 02 days per week.

\subsubsection{Independent Variables}

Independent variables were: age; gender; type of school; area of residence; class attented; person who the adolescent lived with; socio-economic level of parents and the department of residence.

\subsection{Data Analysis}

The data were entered using the Excel software and analysed using the STATA 13.0 software. The qualitative variables were estimated in proportion with their 95\% confidence intervals $(95 \% \mathrm{CI})$ and the quantitative variables were estimated in means followed by \pm standard deviation (SD). Associations between qualitative variables were studied using the Pearson $\mathrm{Chi}^{2}$ test. The strength of the associations between the dependent variables and the independent variables was assessed by calculating Odds Ratios (OR) followed by their $95 \%$ CI. A polytomous logistic regression was used to evaluate the influence of the explanatory variables on the risk factors within our study. All statistically significant variables at a $p$-value $<0.20$ in the univariate analysis were included in the multivariate models and a stepwise descendant modelling procedure was adopted to select the variables that were used to build the final model. Only variables with a p-value of less than 0.05 have been retained in the final model. In order to take into account the effect of the sampling procedure used, all the analyses have been weighted. The adequacy of the model was verified 
using the Hosmer-Lemeshow test.

\subsection{Ethical Consideration}

The protocol was approved by the Institutional Research Ethics Committee of the Institute of Applied Biological Sciences (CER-ISBA). Legally major students (18 years or over) provided their informed consent while the minor ones (less than 18 years) provided their informed assent along with their parents informed consent. All data were anonymous.

\section{Results}

\subsection{Socio-demographic Distribution of Adolescents}

Table 1 and figure 1 shows the distribution of adolescents according to general characteristics. The average age of the 3.968 adolescents included in the study was $16.54 \pm 0.04$ years and the sex ratio male/female was 1.16 . They lived mainly in urban areas (60.5\%) and attended public schools $(68.9 \%)$. More than one in two teens had lived with their parents in the last 30 days preceding the survey.

Table 1. Distribution of school adolescents by socio-demographic characteristics in Benin in 2016.

\begin{tabular}{|c|c|c|}
\hline & Frequencies & Weighted percentage $(\%)$ \\
\hline \multicolumn{3}{|c|}{ Age (Years) $\mathrm{N}=3,968$} \\
\hline$<15$ years & 1,335 & 13.3 \\
\hline$\geq 15$ years & 2,633 & 86.7 \\
\hline \multicolumn{3}{|c|}{ Gender $N=3,953$} \\
\hline Male & 2,127 & 72.9 \\
\hline Female & 1,826 & 27.1 \\
\hline \multicolumn{3}{|c|}{ Areas of residence $\mathrm{N}=3,190$} \\
\hline Rural & 918 & 39.5 \\
\hline Urban & 2,272 & 60.5 \\
\hline \multicolumn{3}{|c|}{ Class attended $\mathrm{N}=3,968$} \\
\hline $1^{\text {st }}$ & 653 & 0.3 \\
\hline $2^{\text {nd }}$ & 794 & 0.2 \\
\hline $3^{\text {rd }}$ & 915 & 30.1 \\
\hline $4^{\text {th }}$ & 657 & 30.7 \\
\hline $5^{\text {th }}$ & 408 & 12.4 \\
\hline $6^{\text {th }}$ & 174 & 11.7 \\
\hline $7^{\text {th }}$ & 367 & 14.6 \\
\hline \multicolumn{3}{|c|}{ Type of school N=3,953 } \\
\hline Public & 2,928 & 68.9 \\
\hline Private & 1,025 & 31.1 \\
\hline \multicolumn{3}{|c|}{ Department $(\mathrm{N}=3,918)$} \\
\hline Alibori & 434 & 17.8 \\
\hline Atacora & 305 & 8.9 \\
\hline
\end{tabular}

\begin{tabular}{lll}
\hline & Frequencies & Weighted percentage (\%) \\
\hline Atlantique & 489 & 9.7 \\
Borgou & 549 & 14.8 \\
Collines & 119 & 4.0 \\
Couffo & 57 & 0.1 \\
Donga & 86 & 2.5 \\
Littoral & 330 & 7.6 \\
Mono & 330 & 7.1 \\
Ouémé & 571 & 11.1 \\
Plateau & 122 & 3.2 \\
Zou & 551 & 12.4 \\
Socio-economic level of parents & \\
Low & 1,356 & 40.1 \\
Average & 987 & 21.5 \\
High & 1,625 & 38.4 \\
\hline
\end{tabular}

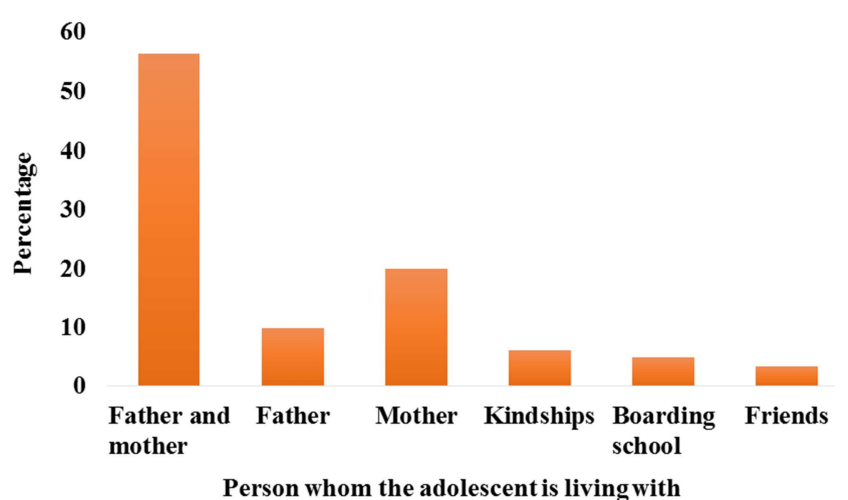

Figure 1. Distribution of school adolescents according to the person who they lived with the last 30 days preceding the survey in Benin in 2016.

\subsection{Prevalence and Factors Associated with NCDs' Risk Factors}

\subsubsection{Prevalence and Factors Associated with Alcohol Consumption Among Adolescents in School Environments in Benin in 2016}

It was $48.3 \%\left(\mathrm{CI}_{95 \%} 0.46-0.50\right)$. The associated factors are summarized in Table 2.

The univariate analysis shows that gender, the class attented and areas of residence were significantly associated with alcohol consumption.

The multivariate analysis shows that the urban areas was protective of alcohol consumption $\left(\mathrm{OR}=0.66 \mathrm{CI}_{95 \%}\right.$ : 0.53 0.83 ) and that there is also an association between the class attented and alcohol consumption.

Table 2. Factors associated with school alcohol consumption among adolescents in Benin in 2016.

\begin{tabular}{|c|c|c|c|}
\hline & \multicolumn{3}{|c|}{ Alcohol consumption } \\
\hline & \multicolumn{2}{|c|}{ Univariate } & \multirow{2}{*}{ Multivariate OR $(95 \%$ CI) } \\
\hline & OR $(95 \%$ CI $)$ & $\mathbf{P}$ & \\
\hline Age & & 0.10 & \\
\hline$<15$ years & 1 & & 1 \\
\hline$\geq 15$ years & $1.22(0.96-1.56)$ & & $0.98(0.73-1.32)$ \\
\hline Gender & & 0.03 & \\
\hline Male & 1 & & 1 \\
\hline Class attended & & 0.01 & \\
\hline 1st & 1 & & 1 \\
\hline 2nd & $2.92(0.70-12.14)$ & & $2.01(0.56-4.15)$ \\
\hline
\end{tabular}




\begin{tabular}{|c|c|c|c|}
\hline & \multicolumn{3}{|l|}{ Alcohol consumption } \\
\hline & \multicolumn{2}{|l|}{ Univariate } & \multirow{2}{*}{ Multivariate OR (95\% CI) } \\
\hline & OR $(95 \%$ CI $)$ & $\mathbf{P}$ & \\
\hline $3 \mathrm{rd}$ & $3.04(1.00-9.21)$ & & $2.06(1.00-4.26)$ \\
\hline 4th & $4.08(1.34-12.40) *$ & & $2.34(1.11-4.96)^{*}$ \\
\hline 5 th & $3.05(0.99-9.33)$ & & $1.91(1,04-4.12)$ \\
\hline 6th & $4.22(1.32-13.41)^{*}$ & & $2.40(1.02-5.65)$ \\
\hline 7th & $3.99(1.30-12.24)^{*}$ & & $2.38(1.11-5.11)^{*}$ \\
\hline Areas of residence & & $10^{-4}$ & \\
\hline Rural & 1 & & 1 \\
\hline Urban & $0.64(0.52-0.79)^{* * *}$ & & $0.66(0.53-0.83)^{* *}$ \\
\hline Department & & 0.0025 & \\
\hline Alibori & 1 & & 1 \\
\hline Atacora & $1.21(0.84-1.76)$ & & 1 \\
\hline Atlantique & $1.21(0.85-1.71)$ & & $1.09(0.72-1.64)$ \\
\hline Borgou & $1.44(1.02-2.05)^{*}$ & & $1.24(0.81-1.89)$ \\
\hline Collines & $1.61(0.93-2.81)$ & & $1.24(0.69-2.23)$ \\
\hline Couffo & $0.38(0.15-0.97)^{*}$ & & $0.37(0.14-0.95)^{*}$ \\
\hline Donga & $0.92(0.48-1.76)$ & & $0.68(0.34-1.36)$ \\
\hline Littoral & $1.57(1.04-2.36)^{*}$ & & $1.32(0.83-2.10)$ \\
\hline Mono & $0.93(0.63-1.37)$ & & $0.82(0.52-1.29)$ \\
\hline Oueme & $1.72(1.24-2.39)^{* *}$ & & $1.49(1.01-20.18)^{*}$ \\
\hline Plateau & $1.79(1.05-3.05)^{*}$ & & $1.49(0.83-2.68)$ \\
\hline Zou & $1.44(1.04-2.00)^{*}$ & & $1.27(0.86-1.88)$ \\
\hline Socio-economic level of parents & & 0.52 & \\
\hline Low & 1 & & \\
\hline Average & $0.91(0.71-1.16)$ & & \\
\hline High & $1.05(0.86-1.28)$ & & \\
\hline Type of school & & 0.5 & \\
\hline Private & 1 & & \\
\hline Public & $1.07(0.87-1.30)$ & & \\
\hline Person who the adolescent most lives with & & 0.87 & \\
\hline Father and mother & 1 & & \\
\hline Father & $1.02(0.74-1.40)$ & & \\
\hline Mother & $1.06(0.84-1.34)$ & & \\
\hline kinships & $1.12(0.76-1.65)$ & & \\
\hline Boarding school & $0.86(0.55-1.33)$ & & \\
\hline Friends & $0.82(0.49-1.39)$ & & \\
\hline
\end{tabular}

$*=\mathrm{p}<0.05 * *=\mathrm{p}<0.01 * * *=\mathrm{p}<0.001$.

\subsubsection{Prevalence and Factors Associated with Tobacco and Cannabis Use Among Adolescents in School \\ Environments in Benin in 2016}

The prevalence of tobacco use was $8 \%\left(\mathrm{CI}_{95 \%}\right.$ 0.06-0.09) and cannabis $2 \%\left(\mathrm{CI}_{95 \%}: 0.01 \%-0.03 \%\right)$.

Table 3 summarizes the factors associated with the prevalence of tobacco and cannabis use. The univariate analysis shows that age; gender; class attented; type of school and areas of residence were significantly associated with tobacco use.

over were about twice as likely to use tobacco. Adolescents in public schools, girls and those living in urban areas have a low probability of using tobacco $\left(\mathrm{OR}=0.29 \mathrm{CI}_{95 \%}\right.$ : 0.16-0.54); $\left(\mathrm{OR}=0.21 \mathrm{CI}_{95 \%}\right.$ : 0.12-0.36); $\left(\mathrm{OR}=0.54 \mathrm{CI}_{95 \%}: 0.33-0.87\right)$.

Cannabis use was significantly associated with gender, the class attented and the person who the adolescent lived with in the univariate analysis.

In the multivariate analysis, girls and adolescents living with their fathers were less likely to use cannabis $(\mathrm{OR}=0.44$ $\mathrm{CI}_{95 \%}$ : 0.22-0.90; $\left.\mathrm{OR}=0.00 \mathrm{CI}_{95 \%}: 0.00-0.00\right)$.

Multivariate analysis shows that adolescents aged 15 and

Table 3. Factors associated with tobacco and cannabis use in school environments among adolescents in Benin in 2016.

\begin{tabular}{|c|c|c|c|c|}
\hline & \multicolumn{2}{|l|}{ Univariate } & \multicolumn{2}{|l|}{ Multivariate } \\
\hline & $\begin{array}{l}\text { Tobacco Consumption OR } \\
(\mathbf{9 5 \%} \mathrm{CI})\end{array}$ & $\begin{array}{l}\text { Cannabis consumption } \\
\text { OR }(95 \% \mathrm{CI})\end{array}$ & $\begin{array}{l}\text { Tobacco Consumption } \\
\text { OR }(95 \% \mathrm{CI}) \\
\end{array}$ & $\begin{array}{l}\text { Cannabis consumption } \\
\text { OR }(95 \% \mathrm{CI})\end{array}$ \\
\hline Age & * & & & \\
\hline$<15$ years & 1 & 1 & 1 & \\
\hline$\geq 15$ years & $1.79(1.20-3.22)$ & $1.44(0.47-4.38)$ & $1.73(1,09--3.88) * *$ & \\
\hline Gender & $* * *$ & * & & \\
\hline Male & 1 & 1 & 1 & 1 \\
\hline Female & $0.21(0.13-0.33) * * *$ & $0.45(0.22-0.93) * *$ & $0.21(0.12-0.36) * * *$ & $0.44(0.22-0.90)^{*}$ \\
\hline Class attended & $* * *$ & * & & \\
\hline $1^{\text {st }}$ & 1 & 1 & 1 & 1 \\
\hline
\end{tabular}




\begin{tabular}{|c|c|c|c|c|}
\hline & \multicolumn{2}{|l|}{ Univariate } & \multicolumn{2}{|l|}{ Multivariate } \\
\hline & $\begin{array}{l}\text { Tobacco Consumption OR } \\
(95 \% \text { CI) }\end{array}$ & $\begin{array}{l}\text { Cannabis consumption } \\
\text { OR }(95 \% \mathrm{CI})\end{array}$ & $\begin{array}{l}\text { Tobacco Consumption } \\
\text { OR }(95 \% \text { CI) }\end{array}$ & $\begin{array}{l}\text { Cannabis consumption } \\
\text { OR }(95 \% \text { CI) }\end{array}$ \\
\hline $2^{\text {nd }}$ & $3.75(1.61-8.73) * *$ & $2.71(0.97-7.60)$ & $1.59(0.26-9.63)$ & $1.63(0.49-5.38)$ \\
\hline $3^{\text {rd }}$ & $5.48(2.64-11.34) * * *$ & $4.11(1.58-10.69) * *$ & $2.24(0.50-9.95)$ & $2.47(0.82-7.40)$ \\
\hline $4^{\text {th }}$ & $6.50(3.12-13.52) * * *$ & $2.85(0.99-8.20)$ & $2.39(0.50-11.27)$ & $1.49(0.46-4.77)$ \\
\hline $5^{\text {th }}$ & $5.73(2.61-12.58) * * *$ & $2.06(0.58-7.29)$ & $3.22(0.63-16.36)$ & $1.16(0.29-4.56)$ \\
\hline $6^{\text {th }}$ & $15.61(6.77-35.97) * * *$ & $7.26(1.77-29.66) * *$ & $3.80(0.71-20.17)$ & $4.12(0.91-18.63)$ \\
\hline $7^{\text {th }}$ & $3.94(1.75-8.88) * * *$ & $4.77(1.68-13.52) * *$ & $1.11(0.23-5.23)$ & $2.62(0.79-8.65)$ \\
\hline Person who the ado lives with & & $* * *$ & & \\
\hline Father and Mother & 1 & 1 & & 1 \\
\hline Father & $0.95(0.50-1.80)$ & $0.00(0.00-0.00 .)^{* * *}$ & & $0.00(0.00-0.00) * *$ \\
\hline Mother & $1.01(0.63-1.63)$ & $1.20(0.52-2.72)$ & & $1.24(0.54-2.86)$ \\
\hline kinships & $0.78(0.34-1.76)$ & $0.12(0.01-0.96)^{*}$ & & $0.12(0.01-0.93)^{*}$ \\
\hline Boarding school & $0.24(0.50-1.02)$ & $1.28(0.28-5.74)$ & & $1.29(0.28-5.79)$ \\
\hline Friends & $1.13(0.46-2.79)$ & 1 & & 1 \\
\hline \multicolumn{5}{|l|}{ Socio-economic level of parents } \\
\hline Low & 1 & 1 & 1 & \\
\hline Average & $0.67(0.37-1.21)$ & $0.56(0.21-1.52)$ & $0.62(0.29-1.28)$ & \\
\hline High & $1.18(0.79-1.75)$ & $1.01(0.45-2.28)$ & $1.28(0.71-2.31)$ & \\
\hline Type of school & $* * *$ & & & \\
\hline Private & 1 & 1 & 1 & \\
\hline Public & $0.50(0.35-0.74) * * *$ & $1.00(0.40-2.45)$ & $0.29(0.16-0.54) * * *$ & \\
\hline Areas of residence & $* *$ & & & \\
\hline Rural & 1 & 1 & 1 & \\
\hline Urban & $0.51(0.33-0.78) * *$ & $0.61(0.27-1.35)$ & $0.54(0.33-0.87) * *$ & \\
\hline Department & $* * *$ & & & \\
\hline Alibori & 1 & 1 & 1 & \\
\hline Atacora & $0.32(0.13-0.80) * *$ & $1.41(0.28-6.91)$ & 1 & \\
\hline Atlantique & $0.89(0.46-1.71)$ & $1.54(0.35-6.73)$ & $1.54(0.50-4.66)$ & \\
\hline Borgou & $1.81(1.02-3.18) * *$ & $2.06(0.45-9.27)$ & $3.26(1.13-9.39) * *$ & \\
\hline Collines & $0.67(0.22-2.02)$ & $3.00(0.50-17.72)$ & $1.97(0.47-8.25)$ & \\
\hline Couffo & 1 & $3.77(0.39-35.68)$ & 1 & \\
\hline Donga & $1.56(0.57-4.27)$ & $4.02(0.69-23.46)$ & $3.39(0.91-12.57)$ & \\
\hline Littoral & $1.69(0.86-3.31)$ & $0.60(0.13-2.80)$ & $5.73(1.89-17.37)^{* *}$ & \\
\hline Mono & $0.23(0.07-0.70) * *$ & $0.70(0.07-6.37)$ & $0.62(0.16-2.39)$ & \\
\hline Oueme & $0.52(0.26-1.06)$ & $1.23(0.31-4.91)$ & $1.83(0.64-5.25)$ & \\
\hline Plateau & $0.86(0.28-2.61)$ & $3.63(0.76-17.29)$ & $3.98(0.95-16.62)$ & \\
\hline Zou & $0.53(0.25-1.11)$ & $2.21(0.62-7.76)$ & $1.16(0.38-3.55)$ & \\
\hline
\end{tabular}

$*=\mathrm{p}<0.05^{* *}=\mathrm{p}<0.01 * * *=\mathrm{p}<0.001$.

\subsubsection{Prevalence and Factors Associated Aux with Insufficient Physical Activity and Physical Inactivity Among Adolescents in Schools in Benin in 2016}

The prevalence of insufficient physical activity was $56.4 \%$ $\left(\mathrm{CI}_{95 \%}: 54.2-58.6 \%\right)$ and that of physical inactivity was $12.4 \%$ ( $\mathrm{IC}_{95 \%}$ : 11.07\%-13.91\%).

Factors associated with low physical activity and with physical inactivity are presented in Table 4.

Gender was associated with insufficient physical activity in the univariate analysis.

In the multivariate analysis, girls were about twice more likely to be insufficiently active than boys $\left(\mathrm{OR}=1,87 ; \mathrm{CI}_{95 \%}\right.$ : $1.57-2.23)$.
Age; gender; class attented; type of school and socioeconomic level of parents were associated with physical inactivity in the univariate analysis.

In the multivariate analysis, adolescents aged 15 and over had a lower risk of being sedentary $\left(\mathrm{OR}=0.65 \mathrm{CI}_{95 \%}\right.$ : 0.460.92 ) compared to younger adolescents. Adolescents with parents with high socio-economic levels were more likely to be sedentary $\left(\mathrm{OR}=1.21 \mathrm{IC}_{95 \%}: 1.04-1.75\right)$ than others. Those attending public schools were less likely to be sedentary $\left(\mathrm{OR}=0.71 \mathrm{IC}_{95 \%}\right.$ : 0.46-0.91) compared with those attending private schools. The class attented was also associated with sedentary lifestyle.

Table 4. Factors associated with physical activity and physical inactivity in schools among adolescents in Benin in 2016.

\begin{tabular}{lllll}
\hline & Univariate & & Multivariate \\
\cline { 2 - 5 } & $\begin{array}{l}\text { Insufficient physical } \\
\text { activity OR (95\% CI) }\end{array}$ & $\begin{array}{l}\text { Sedentary lifestyle } \\
\text { OR (95\% CI) }\end{array}$ & $\begin{array}{l}\text { Insufficient physical } \\
\text { activity OR (95\% CI) }\end{array}$ & $\begin{array}{l}\text { Sedentary lifestyle } \\
\text { OR (95\% CI) }\end{array}$ \\
\hline Age & & $* * *$ & & 1 \\
$<15$ years & 1 & 1 & $0.65(0.46-0.92)^{* *}$ \\
$\geq 15$ years & $0.89(0.70-1.14)$ & $0.47(0.34-0.63) * * *$ & & \\
Gender & $* * *$ & $* *$ & & \\
\hline
\end{tabular}




\begin{tabular}{|c|c|c|c|c|}
\hline & \multicolumn{2}{|l|}{ Univariate } & \multicolumn{2}{|l|}{ Multivariate } \\
\hline & $\begin{array}{l}\text { Insufficient physical } \\
\text { activity OR }(95 \% \text { CI) }\end{array}$ & $\begin{array}{l}\text { Sedentary lifestyle } \\
\text { OR }(95 \% \text { CI })\end{array}$ & $\begin{array}{l}\text { Insufficient physical } \\
\text { activity OR (95\% CI) }\end{array}$ & $\begin{array}{l}\text { Sedentary lifestyle } \\
\text { OR }(95 \% \text { CI })\end{array}$ \\
\hline Male & 1 & 1 & 1 & 1 \\
\hline Female & $1.70(1.44-2.00) * * *$ & $1.44(1.32-1.84)^{* *}$ & $1.87(1.57-2.23) * * *$ & $1.25(0.97-1.62)$ \\
\hline Class attended & & $* * *$ & & \\
\hline $1^{\text {st }}$ & 1 & 1 & 1 & 1 \\
\hline $2^{\text {nd }}$ & $3.38(0.86-13.26)$ & $9.75(2.54-37.39)^{* * *}$ & $1.74(0.40-7.54)$ & $3.34(1.08-10.31)^{* *}$ \\
\hline $3^{\text {rd }}$ & $2.93(1.02-8.45)^{*}$ & $5.35(2.84-10.06)^{* * *}$ & $1.63(0.46-5.80)$ & $2.93(1.66-5.16)^{* * *}$ \\
\hline $4^{\text {th }}$ & $2.25(0.78-6.51)$ & $3.72(1.94-7.13)^{* * *}$ & $1.39(0.39-4.98)$ & $2.28(1.27-4.12)^{* *}$ \\
\hline $5^{\text {th }}$ & $2.80(0.96-8.14)$ & $2.03(0.98-4.19)$ & $1.43(0.40-5.16)$ & $1.25(0.62-2.49)$ \\
\hline $6^{\text {th }}$ & $2.77(0.92-8.41)$ & $2.97(1.27-6.98)^{* *}$ & $1.81(0.48-6.75)$ & $1.56(0.67-3.64)$ \\
\hline $7^{\text {th }}$ & $2.73(0.94-7.96)$ & $2.81(1.40-5.64)^{* *}$ & $2.27(0.63-8.20)$ & $1.94(0.97-3.87)$ \\
\hline Socio-economic level of parents & & * $\quad$ 2 & & \\
\hline Low & 1 & 1 & & 1 \\
\hline Average & $1.18(0.93-1.50)$ & $1.34(0.92-1.93)$ & & $1.07(0.69-1.68)$ \\
\hline High & $0.99(0.81-1.20)$ & $1.35(1.21-1.81)^{* *}$ & & $1.21(1.04-1.75) * *$ \\
\hline Type of school & & $* * *$ & & \\
\hline Private & 1 & 1 & & 1 \\
\hline Public & $1.03(0.85-1.25)$ & $0.63(0.48-0.83)^{* * *}$ & & $0.71(0.46-0, .91)^{* *}$ \\
\hline Department & $* * *$ & $* * *$ & & \\
\hline Alibori & 1 & 1 & 1 & 1 \\
\hline Atacora & $1.23(0.86-1.77)$ & $1.57(0.91-2.71)$ & $1.25(0.54-1.16)$ & $2.05(0.98-4.28)$ \\
\hline Atlantique & $1.48(1.05-2.07)^{* *}$ & $4.36(2.83-6.73)^{* * *}$ & $1.41(0.99-2.00)$ & $4.69(2.81-7.82)^{* * *}$ \\
\hline Borgou & $1.14(0.81-1.61)$ & $1.11(0.63-1.95)$ & $1.13(0.79-1.61)$ & $1.44(0.82-2.54)$ \\
\hline Collines & $0.57(0.32-1.01)$ & $0.85(0.30-2.33)$ & $0.59(0.33-1.04)$ & $1.19(0.38-3.71)$ \\
\hline Couffo & $3.34(1.42-7.89)^{* *}$ & $1.35(0.41-4.44$ & $3.56(1.48-8.59)^{* *}$ & $1.34(0.37-4.80)$ \\
\hline Donga & $0.65(0.33-1.25)$ & $0.00(0.00-0.00)^{* * *}$ & $0.67(0.34-1.30)$ & $0.00(0.00-0.00)^{* * *}$ \\
\hline Littoral & $1.12(0.76-1.67)$ & $1.82(1.05-3.14)^{* *}$ & $1.11(0.74-1.67)$ & $2.25(1.07-4.76)^{* *}$ \\
\hline Mono & $5.01(3.29-7.61)^{* * *}$ & $1.48(0.83-2.61)$ & $6.04(3.93-9.27)^{* * *}$ & $2.15(1.05-4.41)^{* *}$ \\
\hline Oueme & $1.04(0.76-1.44)$ & $1.36(0.62-1.80)$ & $0.97(0.70-1.34)$ & $1.55(0.77-3.13)$ \\
\hline Plateau & $0.33(0.17-0.65)^{* * *}$ & $0.20(0.04-0.87)^{* *}$ & $0.29(0.15-.59)^{* *}$ & $0.33(0.07-1.59)$ \\
\hline Zou & $1.20(0.87-1.64)$ & $0.90(0.53-1.54)$ & $1.27(0.92-1.77)$ & $0.59-2.15)$ \\
\hline \multicolumn{5}{|l|}{ Areas of residence } \\
\hline Rural & 1 & 1 & & \\
\hline Urban & $1.02(0.83-1.25)$ & $1.20(0.89-1.64)$ & & \\
\hline \multicolumn{5}{|l|}{ Person the ado lives with } \\
\hline Father and Mother & 1 & 1 & & \\
\hline Father & $0.88(0.64-1.20)$ & $1.05(0.68-1.62)$ & & \\
\hline Mother & $1.12(0.89-1.41)$ & $1.05(0.76-1.46)$ & & \\
\hline kinships & $1.34(0.91-1.96)$ & $1.05(0.62-1.77)$ & & \\
\hline Boarding school & $0.82(0.53-1.27)$ & $0.67(0.30-1.46)$ & & \\
\hline Friends & $1.30(0.78-2.16)$ & $1.09(0.54-2.18)$ & & \\
\hline
\end{tabular}

$*=\mathrm{p}<0.05 * *=\mathrm{p}<0.01 * * *=\mathrm{p}<0.001$.

\subsubsection{Prevalence and Factors Associated with the Risky Eating Behaviors Among Adolescents in Schools in Benin in 2016}

The prevalence of consumption of fast food (PRR) was $38.6 \%\left(\mathrm{CI}_{95 \%} 36.47-40.75\right)$; the prevalence of consumption of sweet and soft drinks or sodas was $34.9 \%\left(\mathrm{CI}_{95 \%}: 32.8 \%\right.$ $37.0 \%$ ) and the prevalence of insufficient consumption of fruit and vegetables (FEL) was 94.3\% ( $\mathrm{CI}_{95 \%}$ : 93.16\%95.22\%). The factors associated with those variables related to unbalanced diet are summarized in Table 5.

With the univariate analysis:

Age and gender were significantly associated with soda consumption;
Age; class attented; type of school; standard of living and person who the adolescent lived with were significantly associated with the consumption of fast food;

Insufficient consumption of fruit and vegetables is significantly associated with the class attented and gender.

With the multivariate analysis:

Female adolescents were more likely to consume soft drinks than male $\left(\mathrm{OR}=1.39 ; \mathrm{CI}_{95 \%}\right.$ : 1.16-1.67). Adolescents who attended public school were less likely to use them than those who attended private school $\left(\mathrm{OR}=0.71 ; \mathrm{CI}_{95 \%}\right.$ : 0.46-0.91);

Adolescents whose parents had an average socio-economic level were less likely to consume fast food than those whose parents had a low socio-economic level $\left(\mathrm{OR}=0.58 \quad \mathrm{CI}_{95 \%}\right.$ : 0.36-0.94). 
Table 5. Factors associated with the consumption of fast food, sweet and soft drinks (sodas) and insufficient fruit and vegetable consumption (FEL) among adolescents in school places in Benin in 2016.

\begin{tabular}{|c|c|c|c|c|}
\hline \multicolumn{5}{|l|}{ Univariate } \\
\hline & \multirow{2}{*}{$\begin{array}{l}\text { Consumption of sodas OR } \\
(95 \% \text { CI) }\end{array}$} & \multicolumn{2}{|c|}{ Consumption of Fast Food } & \multirow{2}{*}{$\begin{array}{l}\text { Consumption of } \\
\text { insufficient Fruits and } \\
\text { Vegetables OR }(95 \% \mathrm{CI})\end{array}$} \\
\hline & & $\begin{array}{l}1 \text { to } 2 \text { days OR }(95 \% \\
\text { CI) }\end{array}$ & $\begin{array}{l}3 \text { days and more } \mathrm{OR} \\
(95 \% \mathrm{CI})\end{array}$ & \\
\hline Age & **** & ** & & \\
\hline$<15$ years & 1 & 1 & & 1 \\
\hline$\geq 15$ years & $0.59(0.47-0.76) * * *$ & $0.70(0.53-0.91) * *$ & $0.61(0.43-0.88) * *$ & $1.08(0.68-1.72)$ \\
\hline Gender & $* * *$ & & & \\
\hline Male & 1 & 1 & 1 & 1 \\
\hline Female & $1.51(1.27-1.79) * * *$ & $0.97(0.80-1.17)$ & $1.05(0.81-1.37)$ & $0.73(0.52-1.03)$ \\
\hline Class attented & $* * *$ & $* * *$ & & $* *$ \\
\hline $1^{\text {st }}$ & 1 & 1 & & 1 \\
\hline $2^{\text {nd }}$ & $2.75(0.69-10.94)$ & $0.21(0.05-0.78) * *$ & $0.42(0.08-2.19)$ & $0.37(0.17-0.82)^{* *}$ \\
\hline $3^{\text {rd }}$ & $1.84(0.63-5.29)$ & $0.42(0.13-1.35)$ & $0.78(0.17-3.51)$ & $0.37(0.19-0.71)^{* *}$ \\
\hline $4^{\text {th }}$ & $1.77(0.60-5.18)$ & $0.26(0.08-0.83) * *$ & $0.33(0.07-1.51)$ & $0.53(0.26-1.06)$ \\
\hline $5^{\text {th }}$ & $0.72(0.24-2.14)$ & $0.17(0.05-0.55) * *$ & $0.26(0.05-1.20)$ & $0.65(0.30-1.44)$ \\
\hline $6^{\text {th }}$ & $0.96(0.31-2.99)$ & $0.17(0.05-0.60) * *$ & $0.24(0.04-1.22)$ & $0.53(0.19-1.48)$ \\
\hline $7^{\text {th }}$ (Upper Class) & $0.59(0.20-1.77)$ & $0.19(0.06-0.61) * *$ & $0.27(0.06-1.28)$ & $1.480 .59-3.72)$ \\
\hline Socio-economic level of parents & & $* *$ & & \\
\hline Low & 1 & 1 & 1 & 1 \\
\hline Average & $0.81(0.62-1.04)$ & $0.92(0.70-1.21)$ & $0.54(0.36-0.82)$ & $0.89(0.54-1.48)$ \\
\hline High & $0.81(0.661 .00)$ & $0.97(0.77-1.22)$ & $0.89(0.66-1.21)$ & $0.78(0.51-1.21)$ \\
\hline Type of school & & * & & \\
\hline Private & 1 & 1 & 1 & 1 \\
\hline Public & $0.86(0.71-1.06)$ & $0.91(0.73-1.14)$ & $0.66(0.50-0.90)^{* * *}$ & $0.80(0.52-1.24)$ \\
\hline \multicolumn{5}{|l|}{ Areas of residence } \\
\hline Rural & 1 & 1 & 1 & 1 \\
\hline Urban & $1.14(0.92-1.41)$ & $1.03(0.82-1.31)$ & $1.01(0.73-1.40)$ & $0.74(0.47-1.16)$ \\
\hline Person who the ado lives with & & ** & & \\
\hline Father and Mother & 1 & 1 & 1 & 1 \\
\hline Father & $1.16(0.84-1.59)$ & $1.33(0.95-1.87)$ & $1.17(0.72-1.92)$ & $1.00(0.51-1.95)$ \\
\hline Mother & $1.03(0.82-1.31)$ & $0.97(0.74-1.26)$ & $0.89(0.63-1.27)$ & $0.65(0.41-1.02)$ \\
\hline kinships & $0.82(0.55-1.23)$ & $0.95(0.61-1.46)$ & $0.42(0.21-0.86)$ & $1.14(0.51-2.56)$ \\
\hline Boarding school & $1.01(0.64-1.60)$ & $0.47(0.26-0.84)^{*}$ & $0.48(0.23-1.02)$ & $1.84(0.57-6.02)$ \\
\hline Friend & $1.13(0.66-1.94)$ & $0.51(0.27-0.96)^{*}$ & $0.45(0.17-1.21)$ & $1.70(0.65-4.45)$ \\
\hline Department & ** & $* * *$ & & ** \\
\hline Alibori & 1 & 1 & 1 & 1 \\
\hline Atacora & $0.81(0.55-1.20)$ & $1.57(1.03-2.38)^{*}$ & $1.60(0.99-2.59)$ & $1.81(0.72-4.55)$ \\
\hline Atlantique & $0.96(0.67-1.37)$ & $1.24(0.85-1.81)$ & $0.79(0.48-1.32)$ & $1.11(0.54-2.32)$ \\
\hline Borgou & $1.25(0.87-1.78)$ & $0.80(0.53-1.21)$ & $0.43(0.24-0.75) * *$ & $0.60(0.30-1.18)$ \\
\hline Collines & $0.76(0.41-1.44)$ & $0.39(0.18-0.87) *$ & 0 & $0.89(0.28-2.87)$ \\
\hline Couffo & $2.75(1.24-6.09)^{* *}$ & $2.67(1.01-7.01)^{*}$ & $4.68(1.72-12.73)^{* *}$ & $0.36(0.10-1.21)$ \\
\hline Donga & $0.96(0.49-1.88)$ & $3.09(1.54-6.22) * *$ & $1.84(0.73-4.68)$ & $0.56(0.16-1.97)$ \\
\hline Littoral & $0.86(0.57-1.31)$ & $1.34(0.87-2.07)$ & $0.80(0.44-1.45)$ & $3.69(1.13-12.02)^{*}$ \\
\hline Mono & $1.20(0.81-1.77)$ & $0.35(0.21-0.60) * * *$ & $0.13(0.06-0.28)^{* * *}$ & $0.46(0.23-0.94)^{*}$ \\
\hline Oueme & $1.50(1.08-2.08)^{* *}$ & $1.18(0.83-1.70)$ & $0.90(0.56-1.45)$ & $0.53(0.29-0.98)^{*}$ \\
\hline Plateau & $0.51(0.27-0.97)^{*}$ & $0.71(0.38-1.33)$ & $0.18(0.04-0.78)$ & $4.67(0.64-34.18)$ \\
\hline Zou & $1.17(0.84-1.63)$ & $0.60(0.41-0.89) *$ & $0.20(0.10-0.37)$ & $0.66(0.34-1.29)$ \\
\hline
\end{tabular}

Table 5. Continued.

\begin{tabular}{|c|c|c|c|c|}
\hline \multicolumn{5}{|l|}{ Multivariate } \\
\hline & \multirow[b]{2}{*}{$\begin{array}{l}\text { Consumption of } \\
\text { sodas OR }(95 \% \mathrm{CI})\end{array}$} & \multicolumn{2}{|c|}{ Consumption of Fast Food } & \multirow{2}{*}{$\begin{array}{l}\text { Consumption of insufficient } \\
\text { Fruits and Vegetables OR } \\
(95 \% \mathrm{CI})\end{array}$} \\
\hline & & $\begin{array}{l}1 \text { to } 2 \text { days OR }(95 \% \\
\text { CI) }\end{array}$ & $\begin{array}{l}3 \text { days and more OR }(95 \% \\
\text { CI) }\end{array}$ & \\
\hline \multicolumn{5}{|l|}{ Age } \\
\hline$<15$ years & 1 & 1 & 1 & \\
\hline$\geq 15$ years & $0.86(0.65-1.13)$ & $1.06(0.77-1.44)$ & $1.08(0.72-1.61)$ & \\
\hline \multicolumn{5}{|l|}{ Gender } \\
\hline Male & 1 & & & 1 \\
\hline Female & $1.39(1.16-1.67)^{* * *}$ & & & $0,76(0,50-1,16)$ \\
\hline \multicolumn{5}{|l|}{ Class attented } \\
\hline $1^{\text {st }}$ & 1 & 1 & 1 & 1 \\
\hline
\end{tabular}




\begin{tabular}{|c|c|c|c|c|}
\hline \multicolumn{5}{|l|}{ Multivariate } \\
\hline & \multirow{2}{*}{$\begin{array}{l}\text { Consumption of } \\
\text { sodas OR }(95 \% \mathrm{CI})\end{array}$} & \multicolumn{2}{|c|}{ Consumption of Fast Food } & \multirow{2}{*}{$\begin{array}{l}\text { Consumption of insufficient } \\
\text { Fruits and Vegetables OR } \\
\text { (95\% CI) }\end{array}$} \\
\hline & & $\begin{array}{l}1 \text { to } 2 \text { days OR }(95 \% \\
\text { CI) }\end{array}$ & $\begin{array}{l}3 \text { days and more OR }(95 \% \\
\text { CI) }\end{array}$ & \\
\hline $2^{\text {nd }}$ & $1.73(0.36-8.27)$ & $0.20(0.05-0.85)^{*}$ & $0.96(0.24-3.78)$ & $0.86(0.26-2.82)$ \\
\hline $3^{\text {rd }}$ & $1.16(0.34-3.95)$ & $0.40(0.12-1.35)$ & $1.56(0.56-4.38)$ & $0.88(0.31-2.48)$ \\
\hline $4^{\text {th }}$ & $1.18(0.34-4.05)$ & $0.29(0.09-1.01)$ & $0.96(0.34-2.75)$ & $1.03(0.35-3.06)$ \\
\hline $5^{\text {th }}$ & $0.43(0.12-1.52)$ & $0.17(0.05-0.59)^{* *}$ & $0.62(0.21-1.86)$ & $1.69(0.52-5.42)$ \\
\hline $6^{\text {th }}$ & $0.77(0.21-2.80)$ & $0.19(0.05-0.73)^{*}$ & $0.68(0.19-2.51)$ & $1.10(0.26-4.53)$ \\
\hline $7^{\text {th }}$ (Upper Class) & $0.40(0.11-1.41)$ & $0.17(0.05-0.60)^{* *}$ & $0.57(0.19-1.69)$ & $2.73(0.82-9.02)$ \\
\hline \multicolumn{5}{|c|}{ Socio-economic level of parents } \\
\hline Low & 1 & 1 & 1 & \\
\hline Average & $0.87(0.64-1.18)$ & $0.96(0.68-1.36)$ & $0.58(0.36-0.94)^{*}$ & \\
\hline High & $0.89(0.69-1.15)$ & $0.96(0.72-1.28)$ & $1.11(0.74-1.66)$ & \\
\hline \multicolumn{5}{|l|}{ Type of school } \\
\hline Private & 1 & 1 & 1 & \\
\hline Public & $0.62(0.44-0.89) * *$ & $0.83(0.56-1.22)$ & $0.58(0.34-0.98)$ & \\
\hline \multicolumn{5}{|l|}{ Areas of residence } \\
\hline Rural & & & & 1 \\
\hline Urban & & & & $0.91(0.56-1.46)$ \\
\hline \multicolumn{5}{|c|}{ Person who the ado lives with } \\
\hline Father and Mother & & 1 & 1 & 1 \\
\hline Father & & $1.30(0.91-1.85)$ & $1.15(0.68-1.93)$ & $1.29(0.56-2.99)$ \\
\hline Mother & & $1.00(0.76-1.33)$ & $0.94(0.64-1.39)$ & $0.67(0.40-1.11)$ \\
\hline kinships & & $1.06(0.66-1.70)$ & $0.47(0.21-1.02)$ & $1.03(0.44-2.41)$ \\
\hline Boarding school & & $0.74(0.40-1.38)$ & $1.02(0.45-2.32)$ & $1.79(0.54-5.99)$ \\
\hline Friend & & $0.56(0.28-1.10)$ & $0.52(0.18-1.48)$ & $1.39(0.46-4.19)$ \\
\hline \multicolumn{5}{|l|}{ Department } \\
\hline Alibori & 1 & 1 & 1 & 1 \\
\hline Atacora & $1.51(0.88-2.59)$ & $2.05(1.15-3.69)^{*}$ & $2.89(1.40-5.94)^{* *}$ & 1 \\
\hline Atlantique & $1.33(0.86-2.06)$ & $1.47(0.92-2.35)$ & $1.05(0.57-1.93)$ & $0.80(0.29-2.21)$ \\
\hline Borgou & $1.70(1.092 .65)^{* *}$ & $1.02(0.64-1.64)$ & $0.70(0.36-1.38)$ & $0.41(0.16-1.04)$ \\
\hline Collines & $1.60(0.74-3.44)$ & $0.58(0.23-1.45)$ & 0 & $0.42(0.11-1.65)$ \\
\hline Couffo & $3.76(1.56-9.07)^{* *}$ & $2.15(0.75-6.14)$ & $6.55(2.07-20.71)^{*}$ & $0.27(0.06-1.13)$ \\
\hline Donga & $1.91(0.85-4.32)$ & $3.38(1.43-7.98)^{* *}$ & $2.39(0.79-7.19)$ & $0.30(0.72-1.25)$ \\
\hline Littoral & $1.37(0.79-2.36)$ & $1.66(0.90-3.06)$ & $1.62(0.77-3.43)$ & $4.75(1.16-19.52)$ \\
\hline Mono & $2.15(1.26-3.66)^{* *}$ & $0.44(0.23-0.83)^{*}$ & $0.25(0.01-0.66)^{*}$ & $0.26(0.01-0.69)$ \\
\hline Oueme & $2.91(1.78-4.75)^{* * *}$ & $1.57(0.92-2.68)$ & $1.62(0.79-3.33)$ & $0.30(0.12-0.75)$ \\
\hline Plateau & $1.10(0.51-2.36)$ & $1.12(0.52-2.43)$ & $0.42(0.08-2.04)$ & $2.02(0.24-16.67)$ \\
\hline Zou & $(1.17-2.94)^{* *}$ & $0.72(0.43-1.21)$ & $0.29(0.13-0.63)$ & $0.42(0.16-1.05)$ \\
\hline
\end{tabular}

$*=\mathrm{p}<0.05 * *=\mathrm{p}<0.01 * * *=\mathrm{p}<0.001$.

\section{Discussion}

\subsection{Prevalence and Factors Associated with Alcohol Consumption}

The present study shows a prevalence of alcohol consumption of $48.3 \%$. A national study conducted in Argentina by Konfino \& al in 2012 on adolescents aged 13 to 17 in school places in Argentina reported a prevalence of $50.0 \%$, which is close to the one of the present study [8]. Prevalence rates lower than that found in this study were reported by Dagnan N \& al in 2014 in urban areas among secondary school students in Côte d'Ivoire 36.1\% [9]; Gassaye D \& al in 2015 among adolescents in Brazzaville (22.8\%) [10] and Diboh \& al (10.5\%) among students in Côte d'Ivoire in 2013 [11]. On the other hand, other authors have found prevalence rates higher than that observed in the present study. They include Berten \& al (63.4\%) in 2012 among adolescents aged 12 to 18 in schools in Brussels, Belgium [12].

The class attented and areas of residence were associated with alcohol consumption. The association between the class attented and alcohol consumption was also observed by the Quebec National Health Institute in 2015 among young Quebeckers [13]. An association between alcohol consumption and rural areas had already been reported by Cardoso \& al in 2012 in Auvergne [14].

On the other hand, Mimbila-Mayi \& al in 2011 in Gabon found that alcohol consumption varied little according to the adolescent's place of residence [15]. The higher prevalence rate of alcohol consumption in rural areas observed in the present study can be explained by the fact that alcohol is a major ingredient in traditional pharmacopoeia and used in 
many ceremonies.

\subsection{Prevalence Rates and Factors Associated with Tobacco and Cannabis Consumption}

The prevalence rates for tobacco and cannabis consumption were $8 \%$ and $2 \%$ respectively. A prevalence rate of tobacco consumption similar to that reported here was found by Sarr SC \& al en 2007 in Senegal. 7,5\% [16]. Filho $\&$ al in Brazil in 2012 among school adolescents aged 10 to 19 years $(9.3 \%)$ [17] and Befiona \& al found a significantly higher prevalence of $36.3 \%$ among adolescents in school in Madagascar [18]. With regard to cannabis consumption, prevalence rates higher than that found in the present survey have been reported. So Aboobakar \& al in 2011 on the Rodrigues Islands among adolescents aged 13 to 17 years in school had found a prevalence rate of $6.9 \%$ [19]; Berten \& al in Belgium had found a prevalence rate of $11.7 \%$ among $12-$ 18 year olds in schools [12]. The lower smoking prevalence rate observed in the present study, compared with the above cited findings, may be explained by the intensification of tobacco control in schools in Benin.

The low prevalence of cannabis consumption may be explained by the fact that African culture is more stigmatizing to drug use.

Age, gender, type of school attended, areas of residence and the person who the adolescent lived with were associated with tobacco consumption. The male predominance observed in the current survey was reported in most of the studies [14$15,17,20-22]$. This association could be explained by sociocultural factors. Indeed, women smokers are not accepted in African societies.

The male predominance of cannabis consumption observed in this study has already been observed by other authors [15, 23-24].

\subsection{Prevalence Rates and Factors Associated with Insufficient Physical Activity and Sedentary Lifestyle}

The prevalence rate of insufficient physical activity was $56.4 \%$ and the prevalence rate of sedentary lifestyle was 12.4\%. Al-Hazzaa \& al in 2011 in Saudi Arabia among adolescents aged 14 to 19 years has regained a prevalence rate close to that in the present study (44.5\%) [25]. On the other hand, several studies have reported prevalence rates higher than that in the present one [26-28].

This difference could be explained by the insufficient number of hours allocated to physical education and sports classes in schools in Benin and the lack of adequate infrastructure.

Owusu \& al in Ghana in 2008 found a 26.2\% lower prevalence of low physical activity among adolescents in the same age group as the ones in the present survey [22]. The 2009 school-based survey of adolescents aged 13 to 17 years in Benin found a $19 \%$ lower prevalence [7].

Insufficient physical activity was associated with gender. Most studies have shown that girls are less active in physical activity like in the present one [22, 29, 30]. On the other hand, Hamchaoui \& al in 2016 in Algeria among schoolgoing adolescents reached conclusions different from that of the current survey [21].

This difference could be explained in Africa by the fact that girls are forced to do domestic work that is not taken into account as physical activity in their studies. They do not have enough leisure time compared with boys, who are less busy and play games such as football, which are recognized as physical activities.

Variable prevalence rates of sedentary lifestyle have been found in other studies ranging from $11 \%$ to $66.02 \%$ in adolescents [27, 30, 31].

Sedentary lifestyle was associated with age, class attented, socio-economic level of parents and type of school. The association found between age and sedentary lifestyle was also reported by Hamchaoui \& al in 2016 in Algeria [21]. According to those authors, adolescents aged 15 to 19 years and over and those with parents with high socio-economic levels were the most sedentary. Indeed, adolescents with better-off parents have more comfort, cars for travel, electronic devices or gadgets for leisure and would therefore play fewer games requiring physical effort.

\subsection{Prevalence and Factors Associated with Low Fruit and Vegetable Consumption}

In most of the studies consulted, the prevalence of insufficient fruit and vegetable consumption was lower than that observed in the present survey (94.3\%). Indeed, the GSHS study of adolescents aged 13 to 17 in 2009 in Benin reported a prevalence of $81 \%$ [7]. In Ghana, Owusu \& al in 2008 on adolescents aged 13 to 17 years had regained a prevalence of $80 \%$ [22]. An international comparative survey conducted in Europe among adolescents aged 11 to 15 living in France, Denmark and Belgium reported a prevalence of insufficient fruit consumption of $51 \%$ and $45 \%$ for vegetables in 2015 [28]. A similar study conducted by Aboobakar \& al in 2011 on adolescents aged 11 to 17 years found a prevalence of insufficient fruit and vegetable consumption of $72.8 \%$ in Mauritius [19]. Overall, it can be seen that fruit and vegetables are consumed more in some European countries than in Africa. This could be explained by a lack of promotion of fruit and vegetable consumption in African countries.

There are no variables associated with insufficient fruit and vegetable consumption in the present study.

Several authors have found an association between age and insufficient consumption of fruit and vegetables [32-33].

\subsection{Prevalence and Factors Associated with Soft Drink Consumption}

The prevalence of soft drink consumption was $34.9 \%$. Several studies have reported prevalence rates of the same range as in the current survey. National school surveys of adolescents aged 13 to 17 years in Morocco by Gharbi \& al in 2016, in Nepal by Krishna \& al in 2015 and in Mauritius by Aboobakar \& al in 2011 reported prevalence rates of $34 \%$ 
$32 \%$ and $39.7 \%$ respectively $[19,29,30]$. On the other hand, different prevalence rates were reported by Al-Hazzaa \& al (62.35\%) in 2011 among 14-19 year olds in Saudi Arabia [25]; Owusu \& al in 2008 (41.5\%) in Ghana [22] and Benin in $2009(17.6 \%)$ [7].

In the present study, girls were at higher risk of consuming soft drinks. This same association has been corroborated by several authors [19, 34].

\subsection{Prevalence and Factors Associated with the Consumption of Fast Food}

The prevalence of consumption of fast food (PRR) was $38.6 \%$. The studies consulted reported lower prevalence rates than that observed in the present survey. Thus, in Saudi Arabia according to Al-Hazzaa \& al in 2011 the prevalence was $27.5 \%$ [25] and in South Africa Feeley \& al reported in 2009 a prevalence of $30 \%$ among adolescents aged 16 to 18 years [35]. This high prevalence could be explained by the change in dietary behaviour of our populations.

The observed differences can be explained by the difference in the target populations and sites. Indeed, none of those studies focused on adolescents aged from 10 to 19 like the present one and some of them were conducted at the local and regional levels while others were at the national level. The differences could also be explained by the difference in data collection techniques.

\section{Limitations}

For logistical reasons, the study could not be extended to university; this could have led to a selection bias, since adolescents are also at university. However, it is certain that most of adolescents are in secondary schools and very few in universities, which could minimize this selection bias.

Family and genetic factors play a role in the development of some Risk Factors but were not taken into account in the present study.

The self-administration of the questionnaire might have induced some information biases due to interviewees' negligence or misunderstanding of certain questions. However, this situation has been addressed by explaining each question to teens before questionnaire filling. Also, the data may present some desirability bias, as some adolescents may have concealed some bad habits or on the contrary amplified what is perceived as acceptable.

\section{Conclusion}

NCDs represent an imminent "catastrophe", especially for emerging countries. Their behavioural risk factors are increasing as a result of urbanization, globalization and modernization in those countries. The present study revealed that the prevalence of certain risk factors among adolescents in schools in Benin is high. Those high prevalence rates are most often associated with gender, place of residence and socio-economic level depending on the factor studied.
Those results are quite worrying and require NCDs prevention interventions in order to improve adolescent behaviour. Interventions must take into account all the risk factors rather than focusing on a single one, and focus particularly on the early adolescent period and disadvantaged socio-economic groups.

\section{Conflict of Interest}

The authors declare that they have no competing interests.

\section{References}

[1] World Health Organization. Highlights from the WHO Global Conference on NCDs: Enhancing policy coherence to prevent and control noncommunicable diseases 18-20 October 2017, Montevideo, Uruguay [En ligne]. WHO; 2017. Disponible: http://www.who.int/conferences/global-ncd-conference/en/.

[2] Alwan A. Global status report on noncommunicable diseases 2010 [En ligne]. Geneva: WHO; 2010. Disponible: http://www.who.int/nmh/publications/ncd_report2010/en/.

[3] Aubry P, Gaûzère B-A. Les maladies non transmissibles dans les pays du Sud. Médecine tropicale. 2015; 5-7.

[4] Gaimard M. Santé, morbidité et mortalité des populations en développement. Mondes en développement. 2008; (142): 23-38.

[5] Aubry P, Gaüzère B-A. Les cancers dans les pays en développement Actualités 2016. Médecine tropicale. 2016; 7.

[6] Ly A. Enjeux de la prévention des cancers dans les pays du Sud [En ligne]. Grotius International. 2011 Disponible: https://grotius.fr/enjeux-de-la-prevention-des-cancers-dansles-pays-du-sud/.

[7] World Health Organization. Adolescent development [En ligne]. WHO [cité le 9 Sep 2019]. Disponible: https://www.who.int/maternal_child_adolescent/topics/adoles cence $/ \mathrm{dev} / \mathrm{fr} /$.

[8] Konfino J. Global School-based Student Health Survey. Agentina. 2012; 1-2.

[9] N'Cho D, Dagnan SN, Zengbe-Acray P, Ahoussou E-MK, Ekou FK, et al. Consommation d'alcool en milieu urbain chez les élèves du secondaire en Côte d'Ivoire. 2014.

[10] Mabiala-Babela J-R, Mahoungou-Guimbi K-C, Massamba A, Senga $\mathrm{P}$. Consommation de l'alcool chez l'adolescent à Brazzaville (Congo). 2005.

[11] Diboh E, Yao KM, Tako NA, Bakou NF, Assi B. Alcoolisation chez les jeunes élèves en Côte d'Ivoire: préférence et consommation effective. European Scientific Journal. 2013; 9 (30): 380-93.

[12] Berten H, Cardoen D, Brondeel R, Vettenburg N. Alcohol and cannabis use among adolescents in Flemish secondary school in Brussels: effects of type of education. BMC Public Health. 2012 Mar 20; 12: 215.

[13] Tessier S, Hamel D, April N. La consommation excessive d'alcool chez les jeunes Québécois: déterminants et problèmes liés [En ligne]. Québéc: Institut National de Santé Publique du Québec; 2015 p. 36. Disponible: http://www.inspq.qc.ca. 
[14] Cardoso A, Venzac M, Maquinghen S. Drogues et dépendances quelles différences de consommation entre le milieu urbain et le milieu rural? Observatoire Régional de la Santé d'Auvergne; 2012 p. 69.

[15] Mimbila-Mayi M, Vierin Y, Biloghe A, MoussavouMouyama A. Consumption of addictive substances by Gabonese teenagers: An epidemiological survey. Cah d'études Rech Francoph Santé. 2012; 21 (3): 149-52.

[16] Sarr SC, Mane ML, Der D, Dia AA, Diallo K. L'enquête sur le tabac chez les adolescents en milieu scolaire au Sénégal. Sénégal: Ministère de la Sante et de la Prévention; 2007 p. 15.

[17] Barbosa Filho VC, Campos W de, Lopes A da S. Prevalence of alcohol and tobacco use among Brazilian adolescents: a systematic review. Revista de Saúde Pública. 2012 Oct; 46 (5): 901-17.

[18] Befinoana M, Razanamihaja N. Tabagisme et facteurs associés chez les adolescents scolarisés à Madagascar. Sante Publique. 2011; 23 (6): 465-74.

[19] Aboobakar S. GSHS Mauritius Country Report. Mauritius: Ministry of Health \& Quality of Life; 2011.

[20] Nikiéma L, Kouanda S, Seck I, Tiendrebéogo S, Ouédraogo HG, Yaméogo M, et al. Consommation des psychotropes en milieu scolaire, au Burkina Faso: Prévalences et facteurs de risque. Science et Technique, Sciences de la Santé. 1 Jan 2011; 34 (1-2): 65-82.

[21] Hamchaoui F. Besoins de santé et comportements à risque des adolescents scolarisés dans la wilaya d'Alger [Thèse de Médecine]. Alger: Université Alger1 Benyoucef BenkheddaFaculté de Médecine d'Alger; 2016.

[22] Owusu A. Ghana-Global School-based Student Health Survey (GSHS). 2008 p. 100.

[23] Legleye S, Spilka S, Le Nézet O, Hassler C, Choquet M. Alcool, tabac et cannabis à 16 ans-Premiers résultats du volet français de l'enquête ESPAD 2007. 2009; 64: 6.

[24] Estimations 2014 des consommations de produits psychoactifs à 17 ans-Note de synthèse-OFDT [En ligne]. [cité le 9 Sep 2019].

Disponible: https://www.ofdt.fr/publications/collections/notes/estimations2014-des-consommations-de-produits-psychoactifs-17-ans/.

[25] Al-Hazzaa HM, Abahussain NA, Al-Sobayel HI, Qahwaji DM,
Musaiger AO. Physical activity, sedentary behaviors and dietary habits among Saudi adolescents relative to age, gender and region. Int J Behav Nutr Phys Act. 2011 Dec 21; 8: 140-9.

[26] Tremblay MS, LeBlanc AG, Kho ME, Saunders TJ, Larouche R, Colley RC et al. Systematic review of sedentary behaviour and health indicator in school aged childern and youth. Int J Behav Nutr Phys Act. 2011 Sep 21; 8 (98): 5-7. doi: 10.1186/1479-5868-8-98.

[27] Greca JP de A, Silva DAS, Loch MR. Physical activity and screen time in children and adolescents in a medium size town in the South of Brazil. Rev Paul Pediatr. 2016 Jul-Sep; 34 (3): 316-22. doi: 10.1016/j.rppede.2016.01.001.

[28] Kalman M, Inchley J, Sigmundova D, Iannotti RJ, Tynjälä JA, Hamrik $\mathrm{Z}$ et al. Secular trends in moderate-to-vigorous physical activity in 32 countries from 2002 to 2010: a crossnational perspective. Eur J Public Health. 2015 Apr; 25 (Suppl 2): 34-40. doi: 10.1093/eurpub/ckv024.

[29] Gharbi N. GSHS Maroc 2016 Fact Sheet. Morocco: Ministry of Health; 2016.

[30] Krishna A. GSHS Nepal Fact Sheet. Ministry of Health Complex. 2015.

[31] Rapport GSHS Djibouti. Ministères de la Santé, de l'Education Nationale et de l'Enseignement Supérieur, World Health Organisation, Center for Disease Control \& Prevention [En ligne]. 2007 p. $64 . \quad$ Disponible: http://www.who.int/chp/gshs/2007_Djibouti_GSHS_Report.p df?ua $=1$.

[32] Vereecken C, Maes L, De Bacquer D. The influence of parental occupation and the pupils' educational level on lifestyle behaviors among adolescents in Belgium. J Adolesc Health Off Publ Soc Adolesc Med. 2004 Apr; 34 (4): 330-8.

[33] Lytle LA, Varnell S, Murray DM, Story M, Perry C, Birnbaum AS et al. Predicting Adolescents' Intake of Fruits and Vegetables. J Nutr Educ Behav. July-August 2003; 35 (4): $170-8$.

[34] Skinner J, Byun R, Blinkhorn A, Johnson G. Sugary drink consumption and dental caries in New South Wales teenagers. Aust Dent J. 2015 Jun; 60 (2): 169-75.

[35] Feeley A, Pettifor J, Norris S. Fast-food consumption among 17-year-olds in the Birth to Twenty cohort. South Afr J Clin Nutr. 2009; 3 (22): 118-23. 